Z. klin. Chem. u. klin. Biochem.

8. Jg., S. 56-59, Januar 1970

\title{
Die Tagesschwankungen der freien 17-OH-Corticosteroide, Tetrahydroderi- vate, Cortole, Cortolone und der freien polaren Corticosteroide bei Gesunden
}

\author{
Von ANNa Stañ ČÁková
}

Aus dem Wissenschaftlichen Laboratorium der Chirurgiscben Klinik, Universität J. P. Šafárik, Kosice, C.SSR

(Eingegangen am 22. September 1969)

\begin{abstract}
Es wurde die Ausscheidung der freien 17-Hydroxycorticosteroide, der freien polaren Cortiçosteroide, Tetrahydroderivate, Cortole $\left.{ }^{1}\right)$ und Cortolone ${ }^{1}$ ) in zwei Zeiträumen im Verlauf von 24 Stunden verfolgt. Die erste Harnportion wurde von $6-14 \mathrm{Uhr}$ (A), die zweite (B) von 14-6 Uhr früh am nächsten Tag gesammelt. Die Gruppen der Versuchspersonen bildeten 8 Frauen im Alter von $23-45$ Jahren und 10 Männer im Alter von 22-48 Jahren. Es wurde festgestellt, daß die freien, freien polaren und tetrahydrierten Cortisol ${ }^{1}$ )-Metaboliten zu einem Anteil von $60-64 \%$ in die Harnportion A ausgeschieden werden. Das Verhältnis von A/B ist für diese Corticosteroidfraktionen 2,06, 1,96 und 1,85. Die Ausscheidung von Cortolen ${ }^{1}$ ) und Cortolonen ${ }^{1}$ ) ist im Laufe des Tages verzögert und das Verhältnis von $\mathrm{A} / \mathrm{B}$ ist für $\mathrm{C}-17,20,21-$ Glykole $^{1}$ ) im Chloroformextrakt 1,36 und im Äthylacetatextrakt 1,15. Diese Werte sind gegenüber dem Verhältnis von $A / B$ in der Fraktion der freien, freien polaren und tetrahydrierten Corticosteroide deutlich herabgesetzt.
\end{abstract}

\section{The diurnal variation of free 17-bydroxycorticosteroids, tetrabydro-derivatives, cortols, cortolones and the free polar corticosteroids in bealthy individuals}

The excretion of free 17-hydroxycorticosteroids, free polar corticosteroids, tetrahydro-derivatives, cortols ${ }^{1}$ ) and cortolones was measured in two time periods over 24 hours. The first sample (A) was collected between 6 and 14 hours and the second (B) between 14 and 6 hours. The experimental subjects were 8 women aged $23-45$ and 10 men aged $22-48.60-64 \%$ of the free, free polar and tetrahydrogenated cortisol $^{1}$ ) metabolites appeared in sample A. The ratio A/B for the quantities of these corticosteroid fractions was $2.06,1.96$ and 1.85 . During the day, the excretion of cortols and cortolones was retarded and the ratio A/B for C-17,20,21-glycols ${ }^{1}$ ) was 1.36 in chloroform extract and 1.15 in ethyl acetate extract. These $A / B$ values are markedly lower than those for the free, free polar and tetrahydrogenated corticosteroids.

Die rhythmische Konzentrationsänderung von Cortisol $^{1}$ ) und seinen Metaboliten im Blut und im Harn im Verlauf von 24 Stunden stand oftmals im Vordergrund des Interesses (1-11). Thre Bedeutung in der Regulation und im Stoffwechsel von Steroiden unter normalen und pathologischen Bedingungen ist anerkannt. In einer unserer früheren Arbeiten (12) über die Tagesschwankungen der Cortisol $^{1}$ )- und Corticosteron ${ }^{1}$ )-Metaboliten bei Gesunden und Leberkranken registrierten wir für die 17-Hydroxycorticosteroide in der Morgenausscheidung ein Überwiegen der 11-Hydroxy-Metabolite, während der Nacht dagegen der 11-Keto-Metabolite. Das Maximum der Corticosteroidausscheidung lag zwischen 6-12 Uhr.

$\mathrm{Da}$ Cortole ${ }^{1}$ ) und Cortolone ${ }^{1}$ ) nicht nur durch die Reduktion ihrer zugehörigen $\Delta 4-3-$ Ketone, sondern auch durch die Reduktion von THF und THE ${ }^{1}$ ) entstehen $(13,14,15)$, untersuchten wir jetzt, ob die Ausscheidung

1) Trivialnamen und Abkürzungen: Cortisol $=\Delta 4$ Pregnen-11 $\beta, 17 \alpha$ 21-triol-3,20-dion; Cortison $=\Delta 4$-Pregnen-17 $\alpha, 21$-diol-3,11,20trion; Substanz $\mathrm{S}=\Delta 4$-Pregnen-17 $\alpha, 21$-diol-3,20-dion; $\mathrm{THF}=$ $5 \beta$-Pregnan-3 $\alpha, 11 \beta, 17 \alpha, 21$-tetraol-20-on; THE $=5 \beta$-Pregnan$3 \alpha, 17 \alpha, 21$-triol-11,20-dion; A-THF $=5 \alpha$-Pregnan- $3 \alpha, 11 \beta, 17 \alpha, 21$ tetraol-20-on; Cortol $=5 \beta$-Pregnan-3 $\alpha, 11 \beta, 17 \alpha, 20 \alpha, 21$-pentaol; $\beta$-Cortol $=5 \beta$-Pregnan- $3 \alpha, 11 \beta, 17 \alpha, 20 \beta, 21$-pentaol; Cortolon $=5 \beta$ Pregnan- $3 \alpha, 17 \alpha, 20 \alpha, 21$-tetraol-11-on; $\beta$-Cortolon $=5 \beta$-Pregnan$3 \alpha, 17 \alpha, 20 \beta, 21$-tetraol-11-on; Cortole $=$ Cortol $+\beta$-Cortol; Cortolone $=$ Cortolon $+\beta$-Cortolon; $6 \beta-\mathrm{OH}-\mathrm{F}=\Delta 4$-Pregnen- $6 \beta, 11 \beta$, $17 \alpha, 21$-tetraol-3,20-dion; $6 \beta-\mathrm{OH}-\mathrm{E}=\Delta 4$-Pregnen- $6 \beta, 17 \alpha, 21$-triol3,11,20-trion; C-17,20,21-Glykole $=$ Cortole + Cortolone. dieser Cortisol-Metaboliten verzögert ist. Auch $6 \beta$ Hydroxycortisol ist nicht nur als ein echtes Nebennierenrindenprodukt zu betrachten $(16,17)$. Diese Substanz kann im peripheren Gewebe durch $6 \beta$-Hydroxylierung von Cortisol entstehen $(18,19,20)$; aus diesem Grunde könnte ihre Ausscheidung einen verzögerten Verlauf haben. Unsere Ergebnisse bestätigen unsere Annahme im Falle der Cortole und Cortolone, nicht aber im Falle der freien polaren Corticosteroide.

\section{Methodik}

Wir verfolgten die Ausscheidung der Porter-Silber-reagierenden Corticosteroide und C-17,20,21-Glykole im Urin von 8 gesunden Frauen im Alter von 23-45 Jahren und von 10 gesunden Männern im Alter von 22- 48 Jahren. Die Versuchspersonen (Klinikpersonal) sammelten die 24stdg. Urinmenge in zwei Portionen, die erste von 6 Uhr früh bis 14 Uhr nachmittags (Portion A) und die zweite von 14 Uhr bis zu 6 Uhrfrüh am nächsten Tag (Portion B). Die Harnportion A enthielt in jedem Falle den ersten Morgenurin. Der Urin wurde ohne Konservierungsmittel im Kühlschrank aufbewahrt und gleich nach der Sammelperiode verarbeitet. Von beiden Portionen wurde $1 / 4$ bis $1 / 5$ des Volumens auf $\mathrm{pH} 1$ eingestellt und mit Chloroform extrahiert. Dieser Extrakt enthält hauptsächlich freie Corticosteroide. Dann wurde der Urin mit Athylacetat reextrahiert. Dieser Extrakt enthält hauptsächlich freie polare Corticosteroide. Die Harnprobe wurde weiter bei pH 4,5 mit $\beta$-Glucuronidase aus Helix pomatia $46 \mathrm{Stdn}$. inkubiert und wiederholt mit Chloroform und nach Zugabe von 20proz. (w/v) NaCl-Lösung mit Äthylacetat extrahiert. Der erste Extrakt enthält die enzymatisch hydrolysierten Corticoide und der nach Äthylacetatextraktion die enzymatisch hydrolysierten polaten Corticosteroide. Alle Extrakte 
Standardmathoden fitr

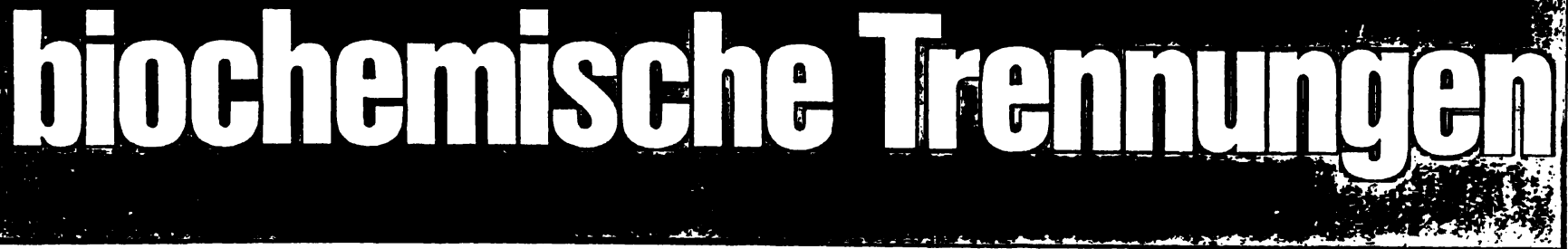

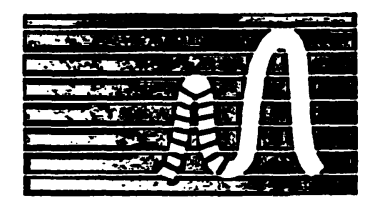

Gelfiltration mit Sephadex und Sepharose

zur Trennung von Molekülen

bis MW $40 \times 10^{6}$. Die Gelfiltra-

tion gestattet Trennungen labiler biologischer Substanzen unter sehr schonenden Bedingungen.

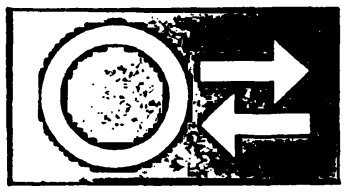

Ionenaustauscher-Chromatographie mit Sephadex-Ionenaustauschern,

die die Vorzüge von CelluloseundKunstharzaustauschern ver-

einigen. Charakteristisch sind hohe Kapazität, niedrige unspezifische Adsorption und ausgezeichnete Reproduzierbarkeit.

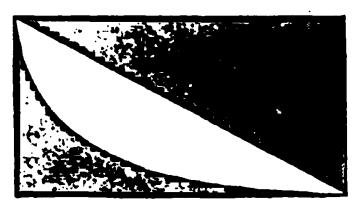

Verteilung in wäßrigen Phasen-Systemen mit Dextran und Dextran-Derivaten

zur Fraktionierung sehr hochmolekularer Stoffe wie Viren, Nukleinsäuren und Zellpartikeln unter sehr milden Bedingungen.

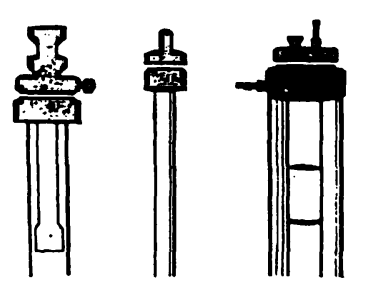

Chromatographierohre.

Unsere Chromatographierohre, die wir speziell für Gelfiltration und lonenaustausch-Chromato-

\section{Literaturdienst}

Als Hilfe für Wissenschaftler geben wir einen umfassenden Literaturdienst heraus. Eine jährlich erscheinende Referenzliste enthält etwa 1000 neue graphie. entwickelt haben, ermöglichen reproduzierbare Trennresultate. Sie stehen Ihnen in großer Auswahl mit diversem Zubehör zur Verfügung.

Literaturstellen. Bitte schreiben Sie uns, wenn wir Sie in unseren Verteiler aufnehmen sollen. Fordern Sie auch Broschüren über unsere Separationsprodukte und das Literaturverzeichnis an. 


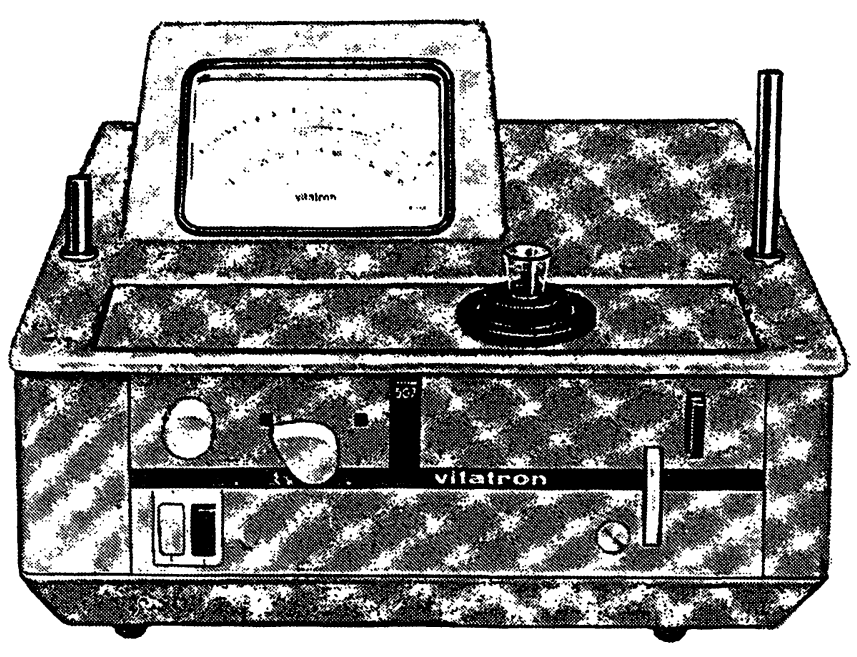

Es hat die Stabilität und Linearität eines

Doppel-Strahl Monochromator-Gerätes, kostet aber weniger als die Hälfte.

Wir können Ihnen hier nicht all seine Vorzüge beschreiben. Aber wenn Sie in Ihrem Labor ein wenig Platz übrig haben (nur $32 \mathrm{~cm} \times 32 \mathrm{~cm}$ ),

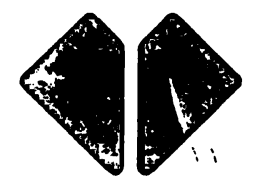

Wir sind sicher:

Das VITATRON UC 200 PRÄZISIONS-COLORIMETER ist eines der stabilsten Ein-Strahl-Photometer der Welt. dann führen wir es Ihnen gern vor. Sollten Sie noch etwas mehr Platz haben ( $30 \mathrm{~cm} \times 43 \mathrm{~cm})$, dann bringen wir auch unseren VITATRON-DIGITALWANDLER mit. Er druckt automatisch die Photometeranzeige als Konzentration oder Extinktion'aus, zusammen mit der Proben-Nummer. Fordern Sie bitte weitere Informationen an! Unsere Service-Station ist in Ihrer Nähe.

\section{wit?atron}

\section{Vitatron $\mathrm{GmbH}$}

5024 Pulheim, Postfach 9 - Ruf: Stommeln $02238 / 7312$

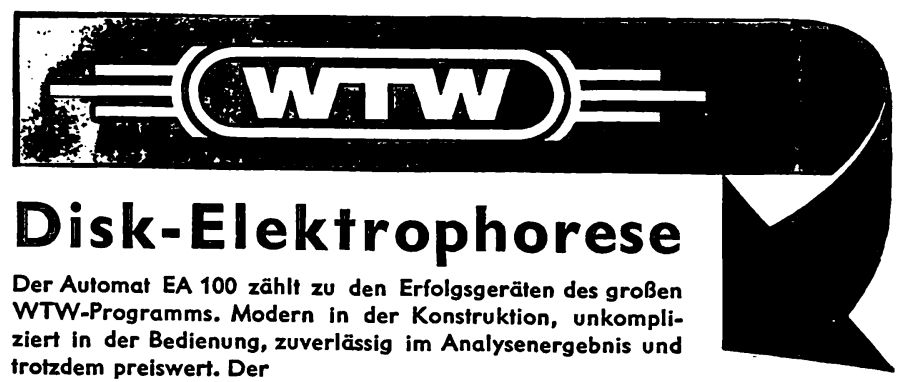

trotzdem preiswert. Der

Disk-Elektrophorese-Automat EA 100 trennt auf der Basis von Polyacrylamidgel mit hervorragenden Trenneigenschaften und kann präparativ oder analytisch betrieben werden. Der Trennvorgang wird durch einfache Programmierung gestevert und das Resultat in getrennten Konzentrationsstufen aufgezeichnet.

Hervorragend bewährt haben sich auch unser Thermoblock TB 4

für die Stickstoffbestimmung biologischer Substanzen, die Ermittlung des gesamten und proteingebundenen Jods und die Blut Blutzuckerbestimmung nach der O-Tolvidin-Methode sowie unsere Zählautomaten für Bakferien-kolonien.

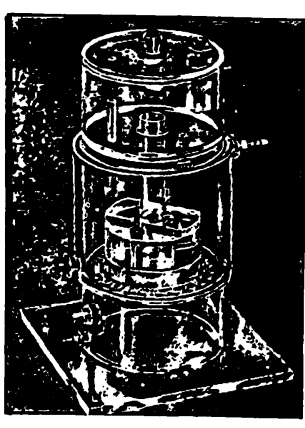

Trennkammer von EA 100
Für ausführliche Informationen stehen Ihr Fachhändler und wir lhnen gern zur Verfügung.

Wissenschaitlich-Technische Werkstäliten GmbH Dr.hab.K.Slevogt · 812 Weilheim · Tel. (0881) 2638/2784

Verkaufbüros: Essen, Lönsberg 22, Tel. 510019

Hagen, Hesterstraße 64, Tel. 45857

Bad Nauheim, Frankfurter Str. 39, Tel. 4860

\section{Kurzgefaßtes} Lehrbuch der

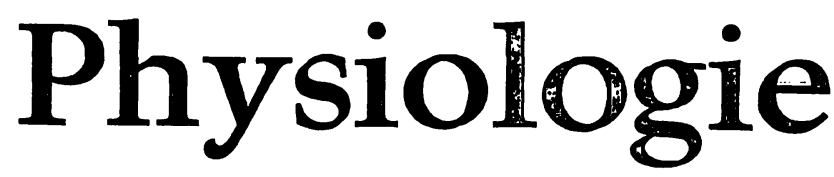

Herausgegeben von

W.D. KEIDEL, Erlangen

Unter Mitarbeit zahlreicher namhafter Fachgelehrter

\section{2., überarbeitete Auflage}

1970. Xll, 524 Seiten, 377 teils farbige Abbildungen in 520 Einzeldarstellungen, 51 Tabellen, Format $17,5 \times 26 \mathrm{~cm}$, PVC kartoniert DM 39,80 (Bestell-Nr. 3586)

Die Neuauflage dieses Buches wurde völlig überarbeitet, wobei auch viele Anregungen aus dem Leserkreis Berücksichtigung fanden. Es erfolgten einige Ergänzungen wie $z$. B. bei dem Kapitel -Gedächtnis und Lernen. und dem Abschnitt über den -Gleichgewichtssinn «.

Durch Hinzunahme neuer Abbildungen, einer teils farbigen Darstellung und Vereinheitlichung sämtlicher Bilder ist eine gestalterisch noch übersichtlichere Anordnung erreicht worden.

\section{Georg Thieme Verlag Stuttgart}


wurden auf einer Silikagel-Kolonne gereinigt und chromatographiert. Die weitere Aufarbeitung wurde an anderer Stelle ausführlich beschrieben (21): Die Extrakte der freien Corticosteroide und der enzymatisch hydrolysierten Corticosteroide wurden mittels Benzol und Athylacetat quantitativ auf eine SilikagelKolonne aufgebracht und stufenweise mit Benzol und steigenden Mengen von Chloroform und Äthylacetat eluiert. Zuletzt wurde die Kolonne mit Methanol eluiert. Im Extrakt der freien Corticosteroide führten wir die Porter-Silber-Reaktion nur in den Eluaten, die Cortison, Substanz S und Cortisol enthielten, durch. Diese Fraktion enthielt auch kleine Mengen von freiem THF und THE. Aus dem Extrakt der enzymatisch hydrolysierten Corticosteroide, in welchem bei der Porter-Silber-Reaktion an erster Stelle THF, A-THF und THE reagieren, wurden die neutralen 17-Ketosteroide durch eine Mischung von Benzol-Chloroform beseitigt. Die C-17,20,21-Glykole wurden im Extrakt der enzymatisch hydrolysierten Corticosteroide nach Oxydation mit Perjodsäure und danachfolgender Ätherextraktion als Zimmermann-reagierende 17-Ketosteroide bestimmt. Diese Farbreaktion wurde in der Relation zur Molekulargewichtsänderung korrigiert. In unseren Arbeitsbedingungen wurde mit Chloroform 73,2 士 $1,2 \% \beta$-Cortolon und $32,7 \pm 1,0 \% \beta$-Cortol extrahiert. In diesen Werten ist auch der Verlust angegeben, der durch Extraktion, Oxydation und Reextraktion mit Ather entsteht. Man kann also den Chloroformextrakt als eine Fraktion, die vorwiegend Cortolone einschließt, betrachten.

Die Extrakte der freien polaren und enzymatisch hydrolysierten polaren Corticosteroide wurden auf die Silikagel-Kolonne mittels einer Mischung von Åthylacetat-Methanol aufgetragen und stufenweise mit Benzol-Äthylacetat, Äthylacetat und Methanol eluiert. Die reine Äthylacetat- und Methanol-Fraktion enthält als Hauptsubstanzen $6 \beta$-OH-F, wir konnten aber in der Mehrzahl der Urine auch $6 \beta-\mathrm{OH}-\mathrm{E}$ nachweisen. Im Extrakt der enzymatisch hydrolysierten polaren Corticosteroide fanden wir mittels Papierchromatographie weitere reduzierende Substanzen, die am Start konzentriert waren. Ihre Identifizierung wurde nicht durchgeführt. Im Extrakt der freien polaren Cortiçosteroide wurde nur die Porter-Silber-Reaktion angewendet. In einem Teil des Extraktes der enzymatisch hydrolysierten polaren Corticosteroide wurde die Porter-Silber-Reaktion durchgeführt, ein weiterer Teil wurde mit Perjodsäure oxydiext und der Gehalt von C-17,20, 21-Glykolen mit der Zimmermann-Reaktion gemessen. Nach Zugabe von reinen Substanzen wurde im Athylacetatextrakt $28,0 \pm 1,2 \% \beta$-Cortolon und $55,3 \pm 1,0 \% \beta$-Cortol wiedergefunden, so daß dieser Extrakt vorwiegend als Cortol-Fraktion zu betrachten ist.

\section{Ergebnisse}

In Tabelle 1 sind die Werte der freien und freien polaren Corticosteroide bei gesunden Frauen und Männern in den Harnportionen $A$ und $B$ verzeichnet. Es ist sichtbar, daß etwa zwei Drittel (63,6 bzw. 63,0\%) der freien Porter-Silber-positiven Corticosteroide in den Morgenund Mittagstunden ausgeschieden werden. Das Verhältnis von A/B ist für die Gruppe der Frauen 2,23, für die Gruppe der Männer 1,90. Keine der Versuchspersonen wies ein Verhältnis von $A / B$ unter 1,00 aus, wir registrierten also in keiner Harnportion $B$ höhere Werte gegenüber der Harnportion A.

Ähnliche Ergebnisse erhielten wir in der Analyse der freien polaren Corticosteroide, die durch Äthylacetat extrahiert werden und vorwiegend durch $6 \beta-O H-F$ repräsentiert sind. Das Verhältnis von $A / B$ ist für die Gruppe der Frauen 2,03, für Männer 1,88. In keinem Falle betrug es unter 1,00.

In Tabelle 2 ist die Ausscheidung der konjugierten Porter-Silber-positiven Corticosteroide bei Gesunden in den Harnportionen A und B verzeichnet. Die Fraktion schließt die Substanzen THF, A-THF, THE und kleine Mengen von THS ein. Das Verhältnis von $A / B$ ist für diese Tetrahydroderivate ähnlich wie für die freien Corticosteroide und beträgt bei den Frauen 1,85 und bei den Männern 1,86. DieWerte der enzymatisch hydrolysierten polaren Corticosteroide sind von dem Verhältnis A/B für die Extrakte der freien, der freien polaren Corticosteroide und für Tetrahydroderivate eindeutig abweichend ( $\mathrm{Tab} .4$ ). Sieben von 18 Versuchspersonen hatten Werte von A/B unter 1,00, d. h. die Ausscheidung dieser Substanzen war in den Nachmittags- und Abendstunden höher.

Die Ausscheidung der mit Chloroform extrahierten C-17,20,21-Glykole, die vorwiegend Cortolone darstellen, zeigt gleichfalls ein niedrigeres Verhältnis von A/B (Tab. 3). Die Werte sind für die Gruppe der Frauen

Tab. 1

Die Ausscheidung der freien (FC) und freien polaren (FPC) Corticosteroide ( $\mu$ g \pm S.D.) in den Harnportionen A und B, der prozentuale Anteil in der Harnportion $A$, das Verhältnis von $A / B$ und die Summe beider (FC + FPC) Werte in 24 Stdn.

\begin{tabular}{|c|c|c|c|c|c|c|c|c|c|}
\hline $\begin{array}{l}\text { Versuchs- } \\
\text { personen }\end{array}$ & A & B & $\stackrel{\%}{A}$ & $A / B$ & A & B & $\stackrel{\%}{\%}$ & $\mathrm{~A} / \mathrm{B}$ & $\underset{\mu \mathrm{g} / 24 \text { Std. }}{\mathrm{FC}}+$ FPC \\
\hline $\begin{array}{l}\text { Frauen } \\
23-45 \mathrm{~J} .\end{array}$ & $\begin{array}{r}265 \\
\pm \quad 83\end{array}$ & $\begin{array}{r}141 \\
\pm \quad 48\end{array}$ & $53,6 \stackrel{63,6}{-86,4}$ & $\begin{array}{r}2,23 \\
+1,69\end{array}$ & $\begin{array}{r}252 \\
+143\end{array}$ & $\begin{array}{r}146 \\
\pm \quad 77\end{array}$ & $50,0^{60,8}-72,4$ & $\begin{array}{r}2,03 \\
\pm 1,25\end{array}$ & $\begin{array}{r}820 \\
\pm 270\end{array}$ \\
\hline $\begin{array}{l}\text { Männer } \\
22-48 \mathrm{~J} .\end{array}$ & $\begin{array}{r}226 \\
\pm 113\end{array}$ & $\begin{array}{r}136 \\
+\quad 84\end{array}$ & $52,0-79,3$ & $\begin{array}{r}1,90 \\
\pm 0,88\end{array}$ & $\begin{array}{r}295 \\
\pm 100\end{array}$ & $\begin{array}{r}178 \\
\pm \quad 88\end{array}$ & $\begin{array}{c}63,0 \\
53,0^{2} \pm 79,0\end{array}$ & $\begin{array}{r}1,88 \\
\pm 0,90\end{array}$ & $\begin{array}{r}840 \\
\pm 290\end{array}$ \\
\hline
\end{tabular}

Tab. 2

Die Ausscheidung der Porter-Silber-positiven Substanzen im Extrakt der enzymatisch hydrolysierten Corticosteroide (EC, P-S posit.) und poDie Ausscheidung der Porter-Silber-positiven Substanzen im Extrakt der enzymatisch hydrontuale Anteil in der Harnportion A, das Verhältnis laren Corticosteroide (EPC, P-S posit.) (mg $\perp$ S.D.) in den Harnportionen A und B, der prozentuale Ante.

\begin{tabular}{|c|c|c|c|c|c|c|c|c|c|}
\hline $\begin{array}{l}\text { Versuchs- } \\
\text { personen }\end{array}$ & \multicolumn{2}{|c|}{$\begin{array}{c}\text { EC } \\
\text { P-S posit. }\end{array}$} & $\begin{array}{l}\% \\
\text { A }\end{array}$ & $A / B$ & \multicolumn{2}{|c|}{$\underset{A}{\stackrel{\text { EPC }}{\text { P-S posit. }} \text { B }}$} & $\begin{array}{l}\% \\
\text { A }\end{array}$ & $\mathrm{A} / \mathrm{B}$ & $\begin{array}{l}E C+\text { EPC } \\
\mathrm{mg} / 24 \mathrm{Stdn}\end{array}$ \\
\hline $23-45 \mathrm{~J}$. & $\begin{array}{r}2,60 \\
\pm 0,60\end{array}$ & $\begin{array}{r}1,52 \\
\pm 0,53\end{array}$ & $58,3^{63,8}-76,5$ & $\begin{array}{r}1,85 \\
\pm 0,55\end{array}$ & $\begin{array}{r}.0,65 \\
\pm 0,20\end{array}$ & $\begin{array}{r}0,63 \\
\pm 0,34\end{array}$ & $37,1 \stackrel{52,5}{-} 73,3$ & $\begin{array}{r}1,26 \\
\pm 0,53\end{array}$ & $\begin{array}{r}5,40 \\
\pm 1,55\end{array}$ \\
\hline $\begin{array}{l}\text { Männer } \\
22-48 \mathrm{~J} \text {. }\end{array}$ & $\begin{array}{r}2,64 \\
\pm 0,64\end{array}$ & $\begin{array}{r}1,60 \\
\pm 0,58\end{array}$ & $55,84,0$ & $\begin{array}{r}1,86 \\
\pm 0,57\end{array}$ & $\begin{array}{r}0,61 \\
\pm 0,31\end{array}$ & $\begin{array}{r}0,57 \\
\text { 上 } 0,23\end{array}$ & $48,4{ }^{53,6}-69,5$ & $\begin{array}{r}1,21 \\
\pm 0,55\end{array}$ & $\begin{array}{r}5,39 \\
\pm 1,33\end{array}$ \\
\hline
\end{tabular}


Tab. 3

Die Ausscheidung der C-17,20,21-Glykole (mg \pm S.D.) im Chloroform(EC)- und Athylacetatextrakt (EPC) in den Harnportionen A und B, der prozentuale Anteil in der Harnportion A, das Verhältnis von A/B und die Gesamtausscheidung dieser Substanzen in 24 Stdn.

\begin{tabular}{|c|c|c|c|c|c|c|c|c|c|}
\hline $\begin{array}{l}\text { Versuchs- } \\
\text { personen }\end{array}$ & \multicolumn{2}{|c|}{$\underset{A}{C-17,20,21-G l y k o l e}$} & & $A / B$ & \multicolumn{2}{|c|}{ 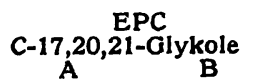 } & $\begin{array}{l}\% \\
\text { A }\end{array}$ & $\mathrm{A} / \mathrm{B}$ & $\begin{array}{l}\text { EC+EPC } \\
\text { Glykole } \\
\text { mg/24 Stdn. }\end{array}$ \\
\hline $345 \mathrm{~J}$ & $\begin{array}{r}1,54 \\
\pm 0,69\end{array}$ & $\begin{array}{r}1,09 \\
\pm 0,26\end{array}$ & $51,3 \frac{57,5}{-71,0}$ & $\begin{array}{r}1,42 \\
\pm 0,51\end{array}$ & $\begin{array}{r}1,42 \\
\pm 0,47\end{array}$ & $\begin{array}{r}1,29 \\
\pm 0,46\end{array}$ & $\begin{array}{c}52,2 \\
44,6-63,2\end{array}$ & $\begin{array}{r}1,12 \\
\pm 0,38\end{array}$ & $\begin{array}{r}5,34 \\
\pm 1,23\end{array}$ \\
\hline $\begin{array}{c}\text { Männer } \\
22-48 \mathrm{~J} .\end{array}$ & $\begin{array}{r}1,24 \\
\pm 0,48\end{array}$ & $\begin{array}{r}0,98 \\
\pm 0,28\end{array}$ & $35,35-64,5$ & $\begin{array}{r}1,33 \\
\pm 0,50\end{array}$ & $\begin{array}{r}1,07 \\
\pm 0,52\end{array}$ & $\begin{array}{r}1,04 \\
\pm 0,54\end{array}$ & $40,83,67,0$ & $\begin{array}{r}1,17 \\
\pm 0,42\end{array}$ & $\begin{array}{r}4,33 \\
+1,25\end{array}$ \\
\hline
\end{tabular}

Tab. 4

Die Verhältnisse von $A / B$ für freie (FC) und freie polare (FPC) sowie enzymatisch hydrolysierte (EC) und enzymatisch hydrolysierte polare (EPC) und C-17,20,21-Glykole in den Extrakten der enzymatisch hydrolysierten Corticosteroide (EC) und enzymatisch hydrolysierten polaren Corticosteroide (EPC) mit Stan

\begin{tabular}{|c|c|c|c|c|c|}
\hline FC & FPC & $\begin{array}{c}\text { EC } \\
\text { P-S posit. }\end{array}$ & $\begin{array}{c}\text { EPC } \\
P \div S \text { posit. }\end{array}$ & $\begin{array}{c}E C \\
\text { C-17,20,21- } \\
\text { Glykole }\end{array}$ & $\begin{array}{c}\text { EPC } \\
\text { C-17,20,21- } \\
\text { Glykole }\end{array}$ \\
\hline \multirow[t]{4}{*}{$\begin{array}{r}2,06 \\
\pm 1,33\end{array}$} & $\begin{array}{r}1,96 \\
\pm 1,04\end{array}$ & $\begin{array}{r}1,85 \\
\pm 0,52\end{array}$ & $\begin{array}{r}1,23 \\
\pm 0,54\end{array}$ & $\begin{array}{r}1,36 \\
\pm 0,49\end{array}$ & $\begin{array}{r}1,15 \\
\pm 0,37\end{array}$ \\
\hline & & & $p<0,02$ & $p<0,05$ & $p<0,01$ \\
\hline & & & $p<0,02$ & $p<0,05$ & $p<0,01$ \\
\hline & $\vdots i$ & & $\mathrm{p}<\underset{\uparrow}{0,02}$ & $\begin{array}{c}p<0,01 \\
\uparrow\end{array}$ & $\begin{array}{c}\mathrm{p}<0,01 \\
\uparrow\end{array}$ \\
\hline
\end{tabular}

1,42 und für Männer 1,33. Zwei von den Versuchspersonen hatten das Verhältnis $A / B$ von weniger als 1,00 . Ein ausgeprägt niedriges Verhältnis von $A / B$ hatten die C-17,20,21-Glykole im Äthylacetatextrakt, die zum größten Teil aus Cortolen bestehen. Für die Frauen war dieses Verhältnis 1,12, für Männer 1,17. Acht von 18 Versuchspersonen wiesen eine höhere Ausscheidung dieser Cortisol-Metaboliten in der Harnportion B auf.

In Tabelle 4 sind die Verhältnisse von $A / B$ aller verfolgten Cortisol-Metaboliten für insgesamt 18 Versuchspersonen verzeichnet. Das Verhältnis von $\mathrm{A} / \mathrm{B}$ ist für die Extrakte der freien und freien polaren Corticosteroide und für die Tetrahydroderivate des Extraktes der enzymatisch hydrolysierten Corticosteriode signifikant unterschiedlich im Vergleich mit dem Verhältnis des Extraktes der enzymatisch hydrolysierten polaren Corticosteroide und den beiden Extrakten von Cortolen und Cortolonen.

\section{Diskussion}

Die Ausscheidung der freien Corticosteroide, die mit Chloroform extrahiert werden, folgt sehr ausgeprägt dem bekannten biologischen Rhythmus und bei keiner von den 18 Versuchspersonen war eine Abweichung sichtbar. Ganz analog verhielten sich die freien polaren Corticosteroide. Die Hauptsubstanz dieser Fraktion, $6 \beta$-Hydroxycortisol hat ihren Ursprung nicht nur in der Nebennierentinde, sie entsteht auch durch $6 \beta$-Hydroxylierung von Cortisol in der Leber, in der Niere und Skelettmuskulatur $(18,19,20)$. Es scheint jedoch, daß die gute Wasserlöslichkeit dieses Steroids seine unmittelbare Ausscheidung zur Folge hat.
Sehr ähnliche Verhältnisse bestehen in der Ausscheidung von THF, A-THF und THE, die in der Form von Glucuroniden zum größten Teil in der Harnportion A $\mathrm{zu}$ finden sind. Abweichende Werte von A/B stellen die Porter-Silber-positiven Substanzen im Extrakt der enzymatisch hydrolysierten polaren Corticosteroide vor. Das Verhältnis ist für beide Gruppen im Durchschnitt 1,23. Wir haben keine Identifizierung dieser Substanzen unternommen, man kann aber vermuten, $\mathrm{da} B$ es sich in diesem Extrakt um hochhydroxylierte Cortisol-Metaboliten handelt, eventuell um Tetrahydro- $6 \beta-\mathrm{OH}-\mathrm{F}$. Auf die Anwesenheit dieses Tetrahydroderivates in menschlichem Urin wiesen ULSTROM und Mitarbeiter (22) und BACCHus (23) hin.

Die Ausscheidung von C-17,20,21-Glykolen ist in beiden Extrakten deutlich verzögert und das Verhältnis von $\mathrm{A} / \mathrm{B}$ ist gegenüber den Steroidfraktionen der freien und freien polaren Corticosteroide und Tetrahydroderivate deutlich herabgesetzt. $\mathrm{Da}$ Cortole und Cortolone hochpolar und deswegen gut wasserlöslich sind, weist ihre verlangsamte Eliminierung aus dem Organismus auf die Tatsache hin, daß sie langsamer als Tetrahydroderivate entstehen und später ausgeschieden werden. Die Nebennierenrinde hat nur eine begrenzte Fähigkeit, die Corticosteroide an C-20 zu reduzieren (24). Die Hauptrolle in dieser Funktion gehört an der ersten Stelle der Leber $(25,26)$, aber auch die Niere $(25,27,28)$ und Skelettmuskulatur $(25,29,30)$ sind an dieser Reduktion in großem Maße beteiligt. $\mathrm{Da}$ es wirklich zu einer extrahepatischen Reduktion von Cortisol kommt, demonstrierte Gold (31), als er C-20 reduzierte Metabolite nach Cortisol-Abgabe bei hepatektomierten Hunden isolierte. Es scheint jedoch, daß die Rolle der Leber in dieser Richtung eine der wichtigsten ist. ZUMOFF und Mitarbeiter (32) und BACCHus (33) konnten bei Patienten mit Lebercirthose ein relatives Ubberwiegen von C-17,20,21-Glykolen gegenüber Porter-Silber-reagierenden Corticosteroiden nachweisen. Es scheint also, daß die Leberschädigung durch veränderte Enzymaktivitäten oder eine reduzierte Durchblutung eine bisher nicht erklärte Erhöhung der C-20 reduzierten Cortisol-Metaboliten zur Folge hat. Unsere Ergebnisse über die tägliche Schwankung der Ausscheidung einzelner Fraktionen der Cortisol-Metabolite zeigt ganz eindeutig, daß Cortole und Cortolone langsamer ausgeschieden werden als freie Corticosteroide und Tetrahydroderivate. Man kann annehmen, daß mindestens ein Teil der C-17,20,21-Glykole sekundär durch den peripheren Stoffwechsel von THF und THE bzw. F und $E$ entsteht und aus diesem Grunde verzögert im Laufe des Tages ausgeschieden wird. 


\section{Literatur}

1. Prncus, G., J. Clin. Endocr. Springfield, 3, 195 (1943). - 2. Pincus, G., L. P. Romanofp und J. Carlo, J. Clin. Endocr. Springfield, 8, 221 (1948). - 3. Bliss, E. L., A. A. SANDbERG, D. H. NeLson und K. EIK-Nes, J. Clin. Invest., 32, 818 (1953). 4. Brown, H., E. Englert, S. Wallach und E. L. Simons, J. Clin. Endocr. Springfield, 17, 1191 (1957). - 5. Forshasx, P. H., V. DiRaimondo, D. Island, A. P. Rinfret und R. H. OrR, Ciba Found. Coll. Endocrinol., 8, 279 (1955). - 6. Lardeaw, J. C., D. JENkins, W. J. Reddy und T. JAcobson, J. Clin. Invest., 33, 950 (1954). - 7. Tyler, F. H., C. Migeon, A. A. Florentin und L. T. Samuels, J. Clin. Endocr. Springfield, 14, 774 (1954). 8. Peterson, R. E., J. Clin. Endocr. Springfield, 17, 1559 (1957). 9. Doe, R. P., E. B. FunNK und M. G. Goodsels, J. Clin. Endocr. Springfield, 16, 196 (1956). - 10. Nugent, Ch. A., K. Erk-Nes, H. S. Kent, L. T. Samuels und F. H. Tyler, J. Clin. Endocr. Springfield, 20, 1259 (1960). - 11. Perkofr, G. T., K. ErK-Nes, C. A. Nugent, H. L. Fred, R. A. Numer, L. Rush, L. T. Samuels und F. H. Truer, J. Clin. Endocr. Springfield, 19, 432 (1959). 12. StančÁkoví, A., Endokrinologie, Leipzig, 44, 49 (1963). 13. Gold, N. I., I. L. Smrth und F. D. MoorE, J. Clin. Invest., 38, 2238 (1959). - 14. BRADLOW, H. L., B. ZuMOFF, T. F. GaILAGHER und L. Hellman, Steroids, 12, 303 (1969). - 15. Zumofr, B., H. L. Bradiow, T. F. Gallaghibr und L. Hellman, J. Clin.
Endocr. Springficld, 28, 1330 (1968). - 16. Burstein, S., R. I. Dorpman und W. M. NAder, Arch. Biochem. Biophysics, 53, 307 (1954). - 17. TOuChs'rone, J. C., M. Kasparow und O. Rosenthul, Fed. Proc., 18, 340 (1959). - 18. Conn, G. L., V. Upton und P. K. Bondy, J. Clin. Findocr. Springfield, 21, 1328 (1961). 19. Lipman, M. M., F. H. Katz und J. W. Jailer, J. Clin. Endocr. Springfield, 22, 268 (1962). - 20. GoLD, N. I., J. Clin. Invest., 41, 1871 (1962). - 21. StančÁkoví, A., Endocr. exper. (Bratislava), im Druck. - 22. Ulstron, R. A., E. Colle, J. Burley und R. Gunville, J. Clin. Endocr. Springfield, 20, 1080 (1960). - 23. Bacchus, H., Clin. Chem. (New York), 13, 855 (1967). - 24. Neher, R. und A. Wettstein, Helv. chim. Acta, 39, 2062 (1956). 25. Mahesh, V. B. und F. Ulrich, J. biol. Chemistry, 235, 356 (1960). 26. Forchielli, E., H. Rosenkrantz und R. I. Dorfaian, J. biol. Chemistry 215, 713 (1955). - 27. De CourCy, C., J. biol. Chemistry, 229, 935 (1957). - 28. GANIS, F. M., L. R. AXELROD und L.L.MILIER, J. biol. Chemistry, 218, 841 (1956). - 29. Thomas, P. Z., E. FORCHIElli und R. I. Dorfman, J. biol. Chemistry, 235, 2797 (1960). 30. De Venuto, F. und U.Westphal, Arch. Biochem. Biophysics, 93 , 423 (1961). - 31. GolD, N.I., J. biol. Chemistry, 236, 1930 (1961). 32. ZuMorf, B., H. L. BradLOW, T. F. Gallagher und L. HellmaN, J. Clin. Invest., 46, 1735 (1967). - 33. Bacchus, H., Clin. Chem. (New York), 15, 237 (1969).

Dr. Ing. Anna Stančáková

Wissenschaftl. Labor. d. Chirurg. Klinik Universität P J Šafárik

Košice, Československo Rastislavova 53 